\title{
Simultaneous Monitoring of Anesthetics and Therapeutic Compounds with a Portable Multichannel Potentiostat
}

\author{
Francesca Stradolini ${ }^{a, *}$, Tamador Elboshra ${ }^{a, b}$, Armando Biscontini ${ }^{a}$, \\ Giovanni De Micheli ${ }^{a}$, Sandro Carrara ${ }^{a}$ \\ ${ }^{a}$ Laboratory of Integrated Systems, Swiss Federal Institute of Technology - Lausanne (EPFL), \\ CH-1015 Lausanne (CH) - * francesca.stradolini@epfl.ch \\ ${ }^{b}$ Department of Electrical and Computer Engineering Khalifa University, United Arab Emirates
}

\begin{abstract}
As metabolic pathways are highly variable among different patients, then an accurate and continuous monitoring of the personal response to drug treatments is essential especially with critical medications, e.g. anesthetic and anti-cancer cocktails. Currently, for anesthesia monitoring, there are no fully mature point-of-care bio-sensing systems. Indeed, it is still evaluated by mathematical models and with indirect parameters like Bispectral Index, which can not provide the actual concentration in blood.

The aim of this paper is to investigate solutions for developing a portable system able to monitor simultaneously several different drugs. For this purpose, Cyclic Voltammetries with an anesthetic product and therapeutic compounds were performed for the validation of the system. A multichannel potentiostat has been realized with Off-The-Shelf Components (COTS). Then, results from electrochemical acquisitions on GC SPEs show that the proposed circuitry is suitable for this aim and they prove its high flexibility to develop portable systems for continuous monitoring of anesthetics and therapeutic compounds.
\end{abstract}

\section{INTRODUCTION}

Personalized therapy offers the opportunity to increase therapeutic efficacy of drugs by targeting the right dosage for each patient and, at the same time, by decreasing toxicity due to overdosing [1]. In this context, Therapeutic Drug Monitoring (TDM) is crucial for difficult-to-manage and critical medications, like anticancer drugs or anesthetics [2]. The aim of this clinical practice is to determine the drug dosage by maintaining the plasma or blood concentration in a targeted therapeutic range to optimize the efficacy in patients' treatments [3].

In case of anesthesia an accurate balanced delivery of several compounds, including anesthetics, analgesics and muscle relaxants is required to achieve a certain target plasma concentration able to assure appropriate sedation and to avoid intoxication or awareness. The effect of anesthesia on patient is expressed as the Depth Of Anesthesia (DOA) which is a very challenging parameter to estimate. Traditional methods to evaluate this crucial parameter are based on the observation of several physiological symptoms such as patient's heart rate and blood pressure [4]. However, these parameters varies a lot depending on the type of surgery [5] and the patient, then they are inadequate [6] and with very poor predictive value [7]. Algorithms based on Bispectral Index (BIS) are actually used in surgery to monitor the DOA of the patient. The BIS is a weighted sum of several electroencephalographic (EEG) parameters [8] and its value decreases linearly with the increasing of the DOA. Unfortunately, also these techniques return wrong estimations in clinical situations due to abnormal EEG patterns or to different anesthetics or to interferences by other drugs [9]. Then, a system able to balance the DOA by administering drugs on the base of their actual concentration in

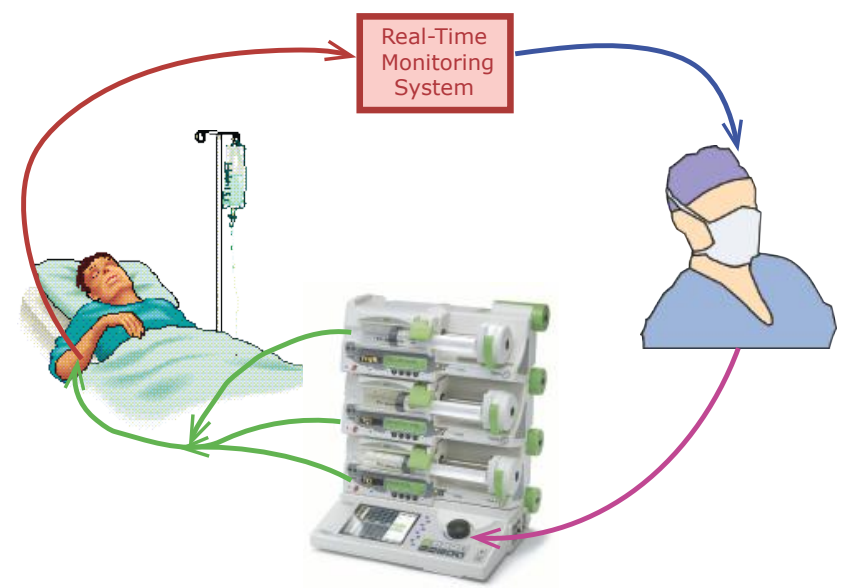

Fig. 1. Conceptual feedback loop system. It consists of three parts: the Target Controlled Infusion (TCI) pump for the anesthesia delivery, the hardware system for continuous monitoring of anesthetics in patient's blood and the anesthesiologist who regulates the infusion pump according to the actual concentration. This closed loop allows the anesthesiologist to have under control the real depth of anesthesia of the patient.

patient's blood could be really attractive for better practice in anesthesiology. The diagram in Fig. 1 presents the concept of a feedback system for anesthesia delivery based on the continuous monitoring of anesthetics in the blood. In this system there are two main sub-systems: the Target-Controlled Infusion (TCI) pump for anesthesia, already largely used in surgical practices, and the real-time monitoring system for the evaluation of anesthetics concentration in patient's blood. The second block allows the anesthesiologist to adjust the infusion rate if necessary, based on real measurements in patient's blood.

Currently, the measurement of drugs concentration in blood is performed using techniques such as mass spectrometry [10], liquid or gas chromatography or immunoassay [11]. However, all of them are not technically suitable for portable online continuous monitoring. Electrochemical biosensors offer, instead, a suitable method for the real-time monitoring in blood since direct electrochemistry does not require the renewal of the electrode surface between measurements meanwhile the selectivity of the electrode could be enhance by the use of a proper membrane [12].

Normally, electrochemical measurement unit setup consists of a sensing part in direct contact with the analyzed solution 


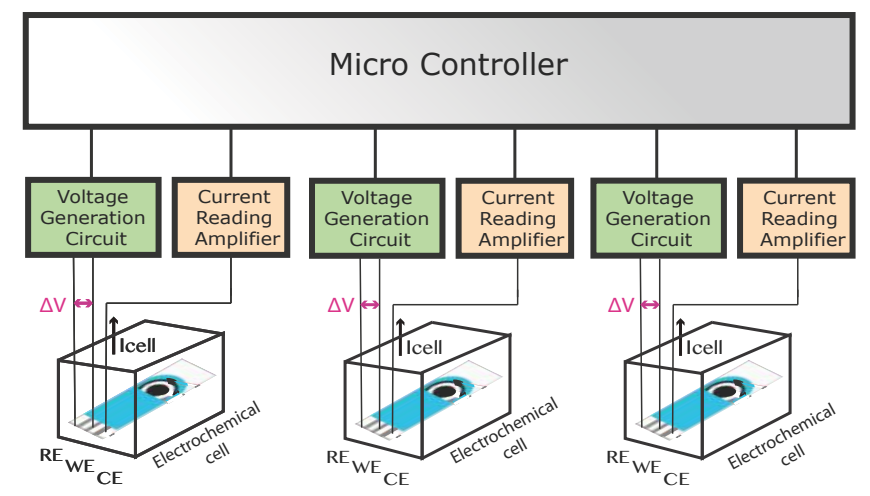

Fig. 2. Sketch of electrochemical cells that interface with the multichannel potentiostat system blocks: micro-controller that drives the voltage generation circuits and receives amperometric values from the readout circuits.

and an electronic part that controls the sensor. The sensing part is usually a three-electrodes (Working, Reference and Counter Electrodes - WE, RE, CE) cell that interfaces with the electronic readout through a potentiostat [13]. Hence, the potentiostat plays a pivotal role by ensuring a constant potential $(\Delta V)$ between RE and WE [14] and by collecting current through $\mathrm{CE}$ so that reduction and oxidation occurs on the surface of WE [15]. The cell potential between RE and WE $(\Delta V)$ is distinct for each analyte, allowing specific compound recognition while the current through $\mathrm{CE}$ permits the quantification of such analyte. Many electrochemical techniques are feasible, but Cyclic Voltammetry (CV) is the most suitable for detecting multiple compounds at the same time [16]. A linear-sweep potential ramp within a specific window range of values is applied for performing a $\mathrm{CV}$ and the output current is continuously monitored.

Over the years, several integrated circuit potentiostats have been developed for chemicals and metabolites detection in [17], [18] but very few are applicable to drugs monitoring [19]. Therefore, more research is required for adopting electrochemical techniques in DOA monitoring. Moreover, a system able to perform simultaneous monitoring of multiple targets is recommended for the several compounds in the anesthetic cocktail.

In this paper we present a new portable system designed to detect several different compounds in a simultaneous way thanks to the independence of the electrochemical cells on the board. We tested the system by performing CVs with an anesthetic, propofol, an analgesic, paracetamol and an anticancer drug, etoposide. It was considered, indeed, the case in which the patient under anesthesia, is also subjected to other therapeutic treatments, which is very common case. The paper is organized as follow: Section II describes the system architecture, Section III collects the procedure and the solutions used for the validation of the system. The validation of the system is presented in Session IV, and, finally, Session $V$ concludes the paper.

\section{SySTEM ARCHITECTURE}

The proposed system, in Fig. 2, consists of three independent electrochemical cells each one in the three-electrodes configuration. The sensing part interfaces with a potentiostat realized using COTS electronic circuit. The circuit, realized on a Printed Circuit Board (PCB) in Fig. 3, also implements three independent amperometric sensing channels with voltage

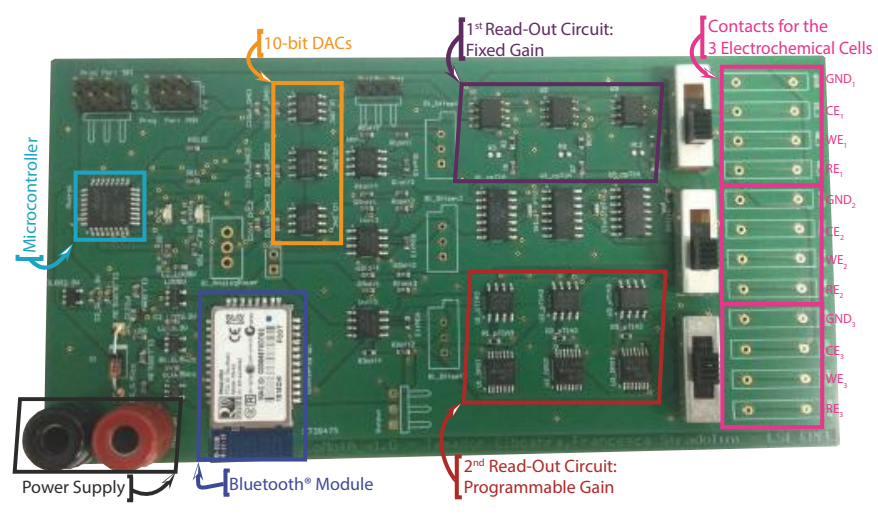

Fig. 3. Printed Circuit Board developed for CV and CA measurements with three independent electrochemical cells.

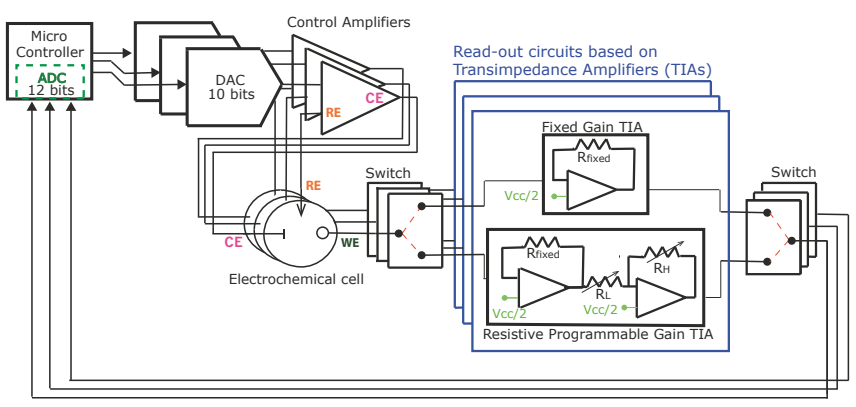

Fig. 4. Detailed architecture of the potentiostatic circuit.

generators and readout circuits for each electrochemical cell. This architecture, then, is able to perform all the measurements with the same front-end enabling the simultaneous monitoring of three different compounds without losing data by switching among them with multiplexing techniques and avoiding any cross-talk through the cells since they are totally independent. The portability of the system is based on small dimensions $16 \times 6 \mathrm{~cm}$ and low power supply $3.3 \mathrm{~V}$, which is reachable by a common battery.

Fig. 4 shows the schematic of the multichannel potentiostat in grounded-WE configuration and arranged for amperometric readout, since the signal which derives from $\mathrm{CV}$ is a current. This current signal derives from the oxidation or reduction of the analyte at the WE surface and it is strictly related to the presence and the concentration of the analyte itself. The basic circuit that realizes the potentiostat features consists of two main parts: (i) the micro-controller (ATxmega32E5 by Atmel AVR XMEGA) that controls the voltage between WE and RE through a 10-bit Digital-to-Analog Coverter (DAC, MCP4911 by Microchip Technology Inc.) and through a control amplifier, and (ii) the transimpedance amplifier (TIA): able to convert the value of the current flowing inside the electrochemical cell into a voltage by the very well-known relation $V_{\text {out }}=-R_{\mathrm{f}} \cdot I_{\text {cell }}$. This analog voltage value is then digitalized by the 12-bit Analog-to-Digital Converter (ADC) of the micro-controller. In particular, the DAC output is buffered with a low precision CMOS amplifier that allows low offset voltage and low noise. The DAC drives capacitive loads without any oscillations, hence, it is suitable for piloting electrochemical cells. Furthermore, the DAC was chosen serial to allow an easier connectivity and programmability. The design of the ADC in the microcontroller depends on pipeline technology which promotes high speed with 12 bit resolution and high 
bandwidth up to $1 \mathrm{MHz}$. Both the voltage generation and the readout circuits are repeated three times to control and read three different electrochemical cells at the same time, as shown in Fig. 4. Therefore, the system can be used to measure simultaneously three sensors by allocating, on the same platform, independent channels for every substance.

\section{A. Voltage generation circuit}

A multiple Direct Digital Synthesizer (DDS) is realized to generate the linear-sweep potential ramps for each electrochemical cell for performing CVs. Each waveform generator is realized by the microcontroller that sends an increasing or decreasing digital value to the specific 10-bit DAC which converts it in an analog voltage. Positive and negative potential values are applied to the WEs thanks to an offset $\left(V_{\mathrm{cc}} / 2\right)$ at the non-inverting input of the TIAs, as shown in Fig. 4. The communication between the microcontroller and the DAC is based on SPI interface. The analog value from the DAC is applied to the sensing cell through a control amplifier that offers high input resistance and zero current suitable for connecting the RE.

\section{B. Different amplifier topologies}

The circuit realizes CV and offers different transimpedance topologies as readouts. To provide a reconfigurable and flexible system, two readout configurations are realized: one TIA with fixed transimpedance gain of $10 k \Omega$ and the other one with two stages of amplification, first stage with fixed transimpedance gain and another amplification stage in cascade. This second stage amplification contains a programmable digital potentiometer TPL0102 by Texas Instruments with 256 wiper positions and an end-to-end resistance of $100 \mathrm{k} \Omega$. Then, the total gain of this second readout circuit will be: $G_{\text {TIA }}=R_{\text {fixed }} \cdot G_{\text {variable }}$ where $\mathrm{R}_{\text {fixed }}$ is $10 \mathrm{k} \Omega$ and $\mathrm{G}_{\text {variable }}$, given by the ratio $R_{\mathrm{H}} / R_{\mathrm{L}}$, is at maximum $100 k$, Fig. 4 . We set the readout configuration of the circuit before starting the measurements, then the characteristics of the switches are negligible.

The flexibility and feasibility of the circuit is given by the separate channels for each analyte so that it is possible to set the suitable gain different for each of them.

When comparing the two readout circuit configurations, the fixed gain TIA results in better signal to noise ratio $(\mathrm{S} / \mathrm{N})$ in comparison with the variable-resistor TIA. This is due to the fact that the digital potentiometer introduces some noise that, added to the noise on the input signal from the sensing cell, is amplified by the second stage.

Finally, it is also important to notice from the schematic in Fig. 4 that, throughout the design, the circuit is virtually grounded at half value of the power supply $\left(\mathrm{V}_{\mathrm{cc}} / 2\right)$ to allow positive and negative voltages therefore implementing a grounded-WE configuration.

\section{MATERIALS AND METHODS}

\section{A. Chemicals}

2,6-Diisopropylphenol (propofol) was purchased from TCI (Switzerland) and prepared first as a $5 \mathrm{mM}$ stock solution in $0.1 \mathrm{M} \mathrm{NaOH}$. Instead, etoposide and paracetamol were both purchased in powder from Sigma-Aldrich (Switzerland). Etoposide $30 \mathrm{mM}$ stock solution was obtained by dissolving $15 \mathrm{mg}$ of powder in $1 \mathrm{ml}$ Dimetil Sulfoxide, also provided by Sigma Aldrich. Finally $5 \mathrm{mg}$ of paracetamol powder were dissolved in $1 \mathrm{ml} 1 \mathrm{x}$ Phosphate Buffered Saline (PBS, pH 7.4) to obtain $30 \mathrm{mM}$ stock solution.

\section{B. Electrochemical Measurements}

Since both the therapeutic compounds and the anesthetic are electroactive, direct CVs were performed in hydrodynamic condition (stirring velocity $140 \mathrm{rpm}$ ) with the here presented circuit. Glassy Carbon (GC) Screen Printed Electrode (SPE) by Methrom (working electrode diameter $4 \mathrm{~mm}$ ) were adopted as electrochemical cells and connected to our PCB for measurements. All experiments were carried out in a $1 \times$ PBS $(\mathrm{pH}$ 7.4) as supporting electrolyte. All the data from measurements has been post-processed with filtering. A Finite Impulse Response (FIR) Low Pass Filter (LPF), with the software Igor Pro 6.03A2, has been applied in order to eliminate the high frequency noise since electrochemical signal is presents only at low frequencies.

\section{System VALIDATION}

Voltammograms reported in Fig. 5 were acquired with the system in a potential range between $-0.8 \mathrm{~V}$ and $+1.7 \mathrm{~V}$ with a scan rate of $100 \mathrm{mV} / \mathrm{s}$ and in stirring conditions (velocity of $140 \mathrm{rpm}$ ). The SPEs, connected to the developed board, were dipped each one in a different $10 \mathrm{ml}$ of $1 \mathrm{x}$ PBS solution ( $\mathrm{pH} 7.4)$, and tested with three different concentrations for each compound. The traces in the charts unequivocally confirm a good performance of the architecture proposed in Fig. 4. Etoposide was tested at $400 \mathrm{mM}, 800 \mathrm{mM}$ and $900 \mathrm{mM}$, propofol at $450 \mathrm{mM}, 500 \mathrm{mM}$ and $530 \mathrm{mM}$, and paracetamol at $300 \mathrm{mM}, 500 \mathrm{mM}$ and $700 \mathrm{mM}$. In each graph characteristic peaks of each analyte can be identified. Moreover, an increase of the traces occurs for each subsequent concentration. It is possible to observe that, in case of propofol, the trace slightly increases between $500 \mathrm{mM}$ and $530 \mathrm{mM}$. This is due to the well known problem of fouling that creates an insulating layer on WE surface which decreases the output signal. This problem is usually solved by adding a PVC membrane on the WE surface, as in [20].

In any case, data compare quite well with those published in literature on the same redox reactions [21], [22], [23].

\section{CONCLUSION}

In this paper, the design, the prototyping and the validation of a portable multichannel potentiostat for the detection of multiple and different drugs is presented. Very different targets, like an anesthetic, propofol, an anti-cancer drug, etoposide and an analgesic, paracetamol, are taken in consideration for proving the flexibility of the circuit. The portability is provided by the small dimensions $(16 \times 6 \mathrm{~cm})$ and low power supply (3.3 V). CVs were performed with the developed circuit using $G C$ SPEs. All the data from electrochemical acquisitions for all the considered analytes have been shown.

More in general, since other anesthetics, such as midazolam and fentanyl are detectable by direct CV [24], [25], this architecture, proved here for propofol monitoring, will be also used for them. Hence, an important step to realize monitoring of anesthetics during surgery and monitoring of therapeutic compounds in Intensive Care Units (ICUs) is achieved.

\section{ACKNOWLEDGMENT}

The authors want to thank the IC-Faculty for the financial support to the student exchange with Khalifa University. The authors also thank Stefano Riario for his technical support. This work was supported by the CoMofA project (\#325230_157139), with grant from the Swiss National Science Foundation. 

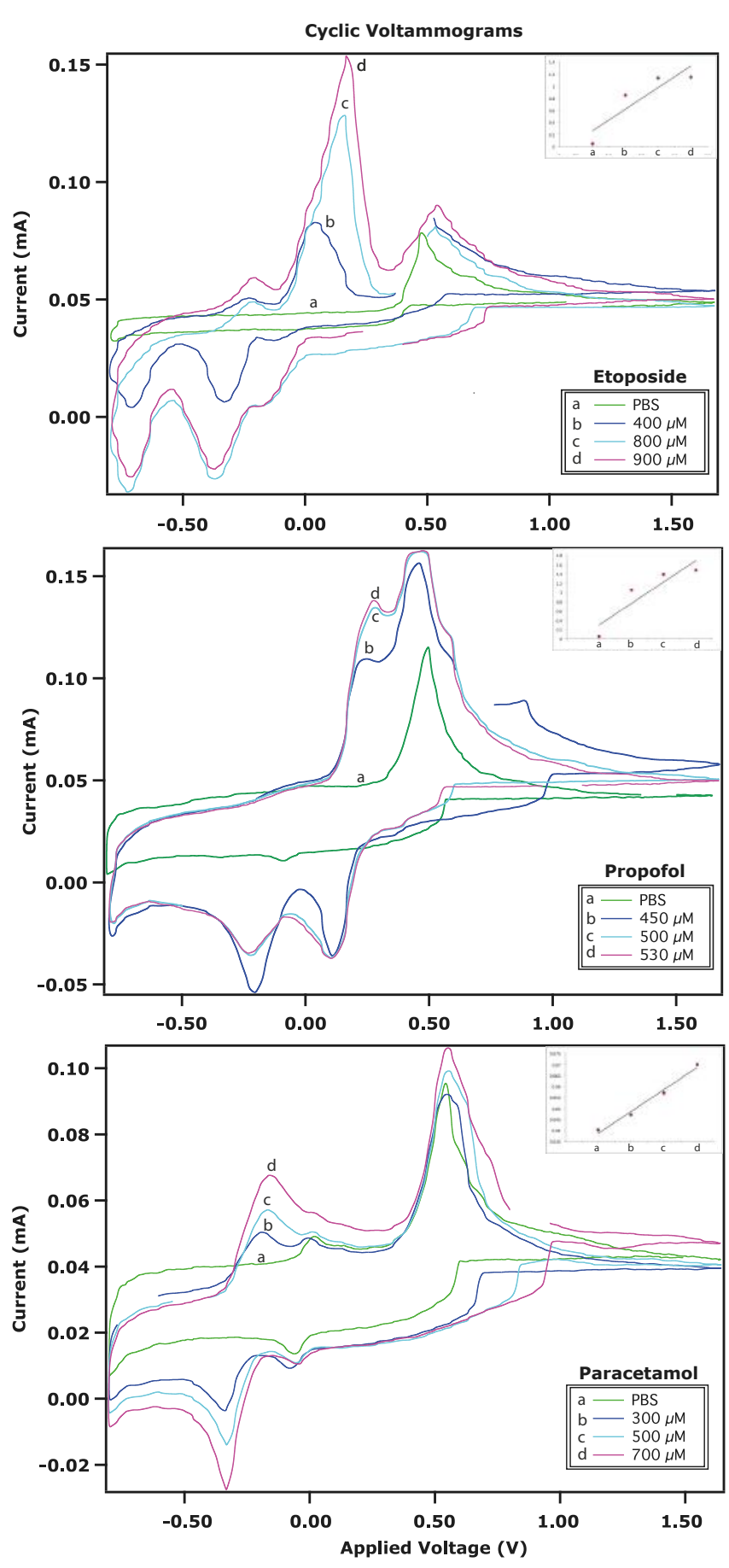

Fig. 5. Cyclic Voltammograms obtained for: etoposide, propofol and paracetamol.

\section{REFERENCES}

[1] A. M. Gonzalez-Angulo, B. T. Hennessy, and G. B. Mills, "Future of personalized medicine in oncology: a systems biology approach," Journal of Clinical Oncology, vol. 28, no. 16, pp. 2777-2783, 2010.

[2] J.-S. Kang and M.-H. Lee, "Overview of therapeutic drug monitoring," The Korean journal of internal medicine, vol. 24, no. 1, pp. 1-10, 2009.

[3] D. Birkett, "Therapeutic drug monitoring," Australian Prescriber, vol. 20, no. 1, pp. 9-11, 1997.

[4] O. Barnea, "Depth of anesthesia monitor," Sep. 12 2000, uS Patent $6,117,075$.
[5] J. F. Talarico, "Therapeutic drug monitoring," Pennsylvania Society of Anesthesiologist, pp. 558-7750, 2012

[6] A. Arslan, B. Sen, F. V. Çelebi, M. Peker, and A. But, "A comparison of different classification algorithms for determining the depth of anesthesia level on a new set of attributes," in Innovations in Intelligent SysTems and Applications (INISTA), 2015 International Symposium on. IEEE, 2015, pp. 1-7.

[7] E. Mortier, M. Struys, T. De Smet, L. Versichelen, and G. Rolly, "Closed-loop controlled administration of propofol using bispectral analysis," Anaesthesia, vol. 53, no. 8, pp. 749-754, 1998.

[8] N. Jagannivas and D. Hepsiba, "Control of anaesthesia concentration using model based controller," in Innovations in Information, Embedded and Communication Systems (ICIIECS), 2015 International Conference on. IEEE, 2015, pp. 1-4.

[9] L. T. D. Duarte and R. Â. Saraiva, "When the bispectral index (bis) can give false results," Revista brasileira de anestesiologia, vol. 59, no. 1, pp. 99-109, 2009.

[10] R. D. Espy, N. E. Manicke, Z. Ouyang, and R. G. Cooks, "Rapid analysis of whole blood by paper spray mass spectrometry for point-ofcare therapeutic drug monitoring," Analyst, vol. 137, no. 10, pp. 23442349, 2012.

[11] S. Basalingappa, A. Sharma, and S. Amarnath, "Basic concepts of therapeutic drug monitoring," Int. J. Current Pharm. Rev. Res, vol. 5, no. 4, pp. 70-75, 2014.

[12] E. Chaum, E. Lindner, K. C. Curley, and J. Guo, "Method and device for detection of bioavailable drug concentration in a fluid sample," Oct. 15 2009, uS Patent App. 13/124,036.

[13] A. J. Bard and L. R. Faulkner, Electrochemical methods: fundamentals and applications. Wiley New York, 1980, vol. 2.

[14] A. Islam, M. Haider, A. Atla, S. Islam, R. Croce, S. Vaddiraju, F. Papadimitrakopoulos, and F. Jain, "A potentiostat circuit for multiple implantable electrochemical sensors," in Electrical and Computer Engineering (ICECE), 2010 International Conference on. IEEE, 2010, pp. 314-317.

[15] M. Duwe and T. Chen, "Low power integrated potentiostat design for $\mu$ electrodes with improved accuracy," in Circuits and Systems (MWSCAS), 2011 IEEE 54th International Midwest Symposium on. IEEE, 2011, pp. 1-4.

[16] S. Carrara, A. Cavallini, V. Erokhin, and G. De Micheli, "Multi-panel drugs detection in human serum for personalized therapy," Biosensors and Bioelectronics, vol. 26, no. 9, pp. 3914-3919, 2011.

[17] A. Mason, Y. Huang, C. Yang, and J. Zhang, "Amperometric readout and electrode array chip for bioelectrochemical sensors," in Circuits and Systems, 2007. ISCAS 2007. IEEE International Symposium on. IEEE, 2007, pp. 3562-3565.

[18] S. S. Ghoreishizadeh, I. Taurino, S. Carrara, and G. De Micheli, "A current-mode potentiostat for multi-target detection tested with different lactate biosensors," in Biomedical Circuits and Systems Conference (BioCAS), 2012 IEEE. IEEE, 2012, pp. 128-131.

[19] S. Ghoreishizadeh, E. G. Kilinc, C. Baj-Rossi, C. Dehollain, S. Carrara, and G. De Micheli, "An implantable bio-micro-system for drug monitoring," in Biomedical Circuits and Systems Conference (BioCAS), 2013 IEEE. IEEE, 2013, pp. 218-221.

[20] F. Kivlehan, F. Garay, J. Guo, E. Chaum, and E. Lindner, "Toward feedback-controlled anesthesia: Voltammetric measurement of propofol (2, 6-diisopropylphenol) in serum-like electrolyte solutions," Analytical chemistry, vol. 84, no. 18, pp. 7670-7676, 2012.

[21] C. Baj-Rossi, G. D. Micheli, and S. Carrara, "Electrochemical detection of anti-breast-cancer agents in human serum by cytochrome p450coated carbon nanotubes," Sensors, vol. 12, no. 5, pp. 6520-6537, 2012.

[22] F. Kivlehan, E. Chaum, and E. Lindner, "Propofol detection and quantification in human blood: the promise of feedback controlled, closed-loop anesthesia," Analyst, vol. 140, no. 1, pp. 98-106, 2015.

[23] M. Tertis, A. Florea, R. Sandulescu, and C. Cristea, "Carbon based electrodes modified with horseradish peroxidase immobilized in conducting polymers for acetaminophen analysis," Sensors, vol. 13, no. 4, pp. 4841-4854, 2013.

[24] R. Jain and R. K. Yadav, "Voltammetric behavior of sedative drug midazolam at glassy carbon electrode in solubilized systems," Journal of Pharmaceutical Analysis, vol. 2, no. 2, pp. 123-129, 2012.

[25] H. Guo, N. Hu, and S. Lin, "Adsorptive stripping voltammetric properties of fentanyl at hg electrode," Talanta, vol. 41, no. 11, pp. 1929-1932, 1994. 